\title{
Actinobacillus sp. Bacteremia in Foals: Clinical Signs and Prognosis
}

\author{
Allison J. Stewart, Kenneth W. Hinchcliff, William J.A. Saville, Eduard Jose-Cunilleras, Joanne Hardy, \\ Catherine W. Kohn, Stephen M. Reed, and Joseph J. Kowalski
}

\begin{abstract}
Medical records of 101 blood culture-confirmed bacteremic foals were reviewed to determine whether foals with Actinobacillus sp. bacteremia are affected at an earlier age, have more severe signs of disease, and have a worse prognosis than do foals with bacteremia of other causes. Thirty percent (30/101) of bacteremic foals had Actinobacillus sp. cultured, and these were 2 times more likely to die (crude odds ratio $\left[\mathrm{OR}_{\mathrm{CR}}\right] 0.8,4 ; P=.14$ ), with a survival rate of $43 \%$ (13/30) compared to the overall survival rate of 55\% (56/101). When compared to other bacteremic foals, foals with actinobacillosis were 7 times more likely to have been sick from birth (adjusted odds ratio $\left.\left[\mathrm{OR}_{\mathrm{ADJ}}\right] 2,26 ; P=.003\right)$ and 6 times more likely to have diarrhea $\left(\mathrm{OR}_{\mathrm{ADJ}} 1,22 ; P=.009\right)$. By bivariate analysis, foals with Actinobacillus sp. bacteremia were 5 times more likely to have a sepsis score $>11\left(\mathrm{OR}_{\mathrm{CR}} 1,18\right.$; $P=.007), 6$ times more likely to be obtunded $\left(\mathrm{OR}_{\mathrm{CR}} 2,20 ; P=.005\right)$, and 3 times more likely to have pneumonia $\left(\mathrm{OR}_{\mathrm{CR}} 1,7\right.$; $P=.03$ ). Furthermore, Actinobacillus sp. bacteremic foals were 27 times more likely to have a segmented neutrophil count $<3.3$ $\times 10^{9}$ cells $/ \mathrm{L}\left(\mathrm{OR}_{\mathrm{ADJ}} 4,166 ; P<.0001\right)$ and were 4.5 times more likely to have a band neutrophil count $>0.46 \times 10^{9}$ cells $/ \mathrm{L}$ $\left(\mathrm{OR}_{\mathrm{ADJ}} 1,17 ; P=.02\right)$ when compared to foals that had bacteremia caused by either gram-negative enteric or gram-positive organisms. Sepsis score was $\leq 11$ in $49 \%$ (29/59) of bacteremia foals aged $<13$ days for which a discernible sepsis score was calculable. Results of this study should improve the diagnostic sensitivity of clinical examinations of neonatal foals, thereby facilitating treatment decisions.
\end{abstract}

Key words: Blood culture; Horses; Neonatal septicemia; Sepsis score.

$\mathbf{I}_{\mathrm{t}}^{\mathrm{i}}$ is unknown if the type of bacterial organism present in the blood of bacteremic foals influences presenting clinical signs or subsequent prognosis. The most common etiologic agents isolated from bacteremic foals are gram-negative enteric bacteria, such as Enterobacteriaceae, and Actinobacillus sp. Escherichia coli is generally the most frequently isolated organism from the blood of bacteremic equine neonates, ${ }^{1-7}$ with Actinobacillus sp. often identified as the 2nd most common isolate. ${ }^{2,4,8}$ Actinobacilli are pleomorphic gram-negative rods and appear to be distinct from enteric Enterobacteriaceae in their antimicrobial susceptibility patterns, ${ }^{5,9}$ and anecdotally in their presentation of clinical disease. Several species of Actinobacillus have been associated with infection in the horse, with $A$. equuli being the best documented. Although discrepancy in identification exists, "A. suis-like" and other undifferentiated actinobacilli also have been recognized as equine pathogens. ${ }^{10,11} \mathrm{Al}$ though Actinobacillus sp. frequently are cultured from the blood of foals, the relative importance of actinobacillosis in foals has, to our knowledge, not been formally investigated.

Historically, A. equuli neonatal bacteremia is reported to

From the Department of Veterinary Clinical Sciences (Stewart, Hinchcliff, Jose-Cunilleras, Hardy, Kohn, Reed, Kowalski), and the Department of Veterinary Preventive Medicine (Saville), The Ohio State University, Columbus, OH. Sections of this study were presented previously as a poster at the 15th Edward F. Hayes Graduate Research Forum, Ohio State University, Columbus, OH, 2001, as a research abstract at The 19th ACVIM meeting, Denver, 2001, and as an abstract at the 3rd Dorothy Russell Havemeyer Foundation Neonatal Septicemia workshop, Talloires, France, 2001.

Reprint requests: Kenneth W. Hinchcliff, BVSc, PhD, Department of Veterinary Clinical Sciences, College of Veterinary Medicine, The Ohio State University, 601 Tharp Street, Columbus, OH 43210; e-mail: hinchcliff.2@osu.edu.

Submitted September 24, 2001; Revised November 27, 2001; Accepted January 21, 2002.

Copyright (C) 2002 by the American College of Veterinary Internal Medicine

0891-6640/02/1604-0000/\$3.00/0 lead to an acute, highly fatal septicemia of newborn foals. The syndrome previously was known as shigellosis (Shigella equirulis) or colloquially as "sleepy foal" disease. Actinobacillus sp. bacteremia was reported to affect foals within a few hours of birth up to 3 days of age. It is associated with sudden onset of fever, prostration, and diarrhea and frequently localization of the organism to joints, kidneys, lungs, and intestine. The disease commonly results in death of affected foals..$^{3,5,12-16}$

Based on anecdotal reports, we hypothesized that foals with Actinobacillus sp. bacteremia succumb to infection at an earlier age, have an increased occurence of failure of transfer of passive immunity, and have a decreased survival rate. ${ }^{3,5,6,17-19}$ The purpose of this retrospective study was to determine outcome and indicators of disease severity in foals with Actinobacillus sp. bacteremia, and to identify diagnostic factors that differentiate foals with actinobacillosis from foals with bacteremia caused by either enteric gramnegative or gram-positive organisms.

\section{Materials and Methods}

A retrospective observational study was performed to determine the factors specifically associated with actinobacillosis in foals. All blood culture-confirmed bacteremic foals admitted to The Ohio State University Veterinary Teaching Hospital from 1993 through 2000 were eligible for enrollment in the study. Aerobic and anaerobic cultures were routinely performed on blood obtained from an aseptically prepared jugular vein on the day of admission from all foals in which septicemia was suspected, regardless of age or previous antibiotic therapy. The medical records of 102 foals were reviewed. One bacteremic foal with a hepatoblastoma was excluded from the study because of its inevitable death from the congenital defect.

\section{Collection of Data}

Information obtained from medical records included signalment (year of admission, age of foal at admission [hours], and breed) and historical information obtained from the owner or agent (dystocia or delivery from a sick mare, duration of illness [hours], evidence of illness from birth, failure to stand after birth, ability to nurse the mare, and ingestion of colostrum [nursed from the mare, a bottle, or admin- 
istered by nasogastric tube]). Information recorded from the initial physical examination at presentation included rectal temperature, heart rate, respiratory rate, weight, ability to stand, mentation (categorized as bright, depressed, or comatose), presence of seizures, presence of a suckle reflex, hydration status (normal, $<8 \%$ dehydrated, and $\geq 8 \%$ dehydrated), presence of diarrhea, presence of colic, or presence of joint distention. Venous and arterial blood gas analysis $(\mathrm{pH}$, partial pressure of carbon dioxide $\left[\mathrm{PCO}_{2}\right]$, partial pressure of oxygen $\left[\mathrm{PO}_{2}\right]$, bicarbonate, and base excess), hematologic data (PCV, hemoglobin concentration, total white blood cell count, segmented neutrophil, band neutrophil, lymphocyte, monocyte, and platelet concentrations), biochemical findings (total $\mathrm{CO}_{2}$, total calcium, phosphorus, sodium, chloride, potassium, glucose, creatinine, serum urea nitrogen, total protein, fibrinogen and albumin concentrations and activity of alkaline phosphatase [ALP], creatine kinase, aspartate aminotransferase, gammaglutamyl transferase, and sorbitol dehydrogenase), and immunoglobulin $\mathrm{G}(\mathrm{IgG})$ concentration (categorized as 0-200 mg/dL, 200-400 $\mathrm{mg} / \mathrm{dL}, 400-800 \mathrm{mg} / \mathrm{dL}$, and $>800 \mathrm{mg} / \mathrm{dL}$ ) were obtained at the initial examination. A sepsis score was calculated from the data as described by Brewer and Koterba. ${ }^{20}$ The presence of pneumonia indicated by thoracic radiography or confirmed at postmortem examination, evidence of septic arthritis (clinical or postmortem diagnosis), survival to discharge ( 2 foals that were discharged because of financial constraints and died 24-48 hours later were considered to have died at the hospital for analysis purposes), and duration of hospitalization to discharge for surviving foals were recorded.

\section{Laboratory and Clinical Methods}

Blood samples for culture were obtained after clipping and shaving the hair, aseptic preparation of the skin, and removal of $20-40 \mathrm{~mL}$ of blood into a sterile syringe from a jugular vein. The sampling needle then was discarded and separate needles were used to inoculate $10 \mathrm{~mL}$ of blood into each of the commercially available aerobic and anaerobic culture bottles. ${ }^{a}$ Routine microbiology then was performed by The Ohio State University Veterinary Microbiology Laboratory.

For routine biochemical and hematologic analysis, blood either was collected from a large peripheral vein (cephalic, femoral, or saphenous) or from the jugular vein at the time of catheter placement. Blood was placed into evacuated glass tubes ${ }^{\mathrm{b}}$ containing no anticoagulant for serum biochemistry and immunoglobulin quantification, ethylenediaminetetraacetic acid for hematology, and sodium citrate for fibrinogen concentration. Blood also was collected anaerobically into 3-mL plastic syringes, the dead space of which was filled with sodium heparin $(1,000 \mathrm{IU} / \mathrm{mL})$ for blood gas analysis. Venous samples were collected as described above, and arterial samples were collected from either the dorsal metatarsal or the facial artery. Hemograms were performed with a commercial semiautomated multichannel blood cell-counting system, ${ }^{c}$ with differential counts performed by manual cytologic examination of a blood smear. Serum biochemistry was performed with a commercial automated analyzer. ${ }^{\mathrm{d}}$ Immunoglobulin concentration was determined semiquantitatively with a commercially available concentration immunoassay technology test. ${ }^{e}$ Fibrinogen concentrations were measured with a commercial automated analyzer. ${ }^{\mathrm{f}}$ Arterial and venous blood $\mathrm{pH}, \mathrm{PCO}_{2}$, and $\mathrm{PO}_{2}$ were measured and base excess was calculated within 15 minutes of blood collection with an automated blood gas analyzer. ${ }^{\mathrm{g}}$ Blood gas tensions and $\mathrm{pH}$ were not corrected for divergence of the temperature of the analyzer $\left(37^{\circ} \mathrm{C}\right)$ from that of the foal.

\section{Statistical Analysis}

Data from the entire population of foals were summarized by calculating standard descriptive statistics. Frequency distributions of categorical variables were evaluated, and means, medians, standard deviations, and ranges were calculated, as appropriate, for continuous variables.
Three non-mutually exclusive categories then were formed based on the type of microorganism cultured from the blood: foals from which Actinobacillus sp. (A. equuli and A. suis) were isolated; foals from which gram-negative enteric bacteria (Pseudomonas sp. and genera of Enterobacteriaceae such as Escherichia coli, Klebsiella sp., Proteus sp., Enterobacter sp., and Salmonella sp.) were cultured; and foals that had blood culture growth of gram-positive organisms (Streptococcus sp., Staphylococcus sp., Enterococcus sp., and Listeria monocytogenes).

Continuous variables were categorized to facilitate logistic regression analysis, with divisions made above and below the median value calculated from the entire population of foals for each variable, except for rectal temperature (categorized as $<100^{\circ} \mathrm{F}\left[<37.8^{\circ} \mathrm{C}\right], 100-102^{\circ} \mathrm{F}$ $\left[37.8-38.9^{\circ} \mathrm{C}\right]$, and $>102^{\circ} \mathrm{F}\left[>38.9^{\circ} \mathrm{C}\right]$ ), creatinine (categorized as $>2.5 \mathrm{mg} / \mathrm{dL}$ or $<2.5 \mathrm{mg} / \mathrm{dL}$ ), fibrinogen (categorized as $>400 \mathrm{mg} / \mathrm{dL}$ or $<400 \mathrm{mg} / \mathrm{dL}$ ), IgG (categorized as $<400 \mathrm{mg} / \mathrm{dL}$ or $>800 \mathrm{mg} / \mathrm{dL}$ ), and sepsis score (categorized as $\leq 11$ or $>11$ ). All independent variables initially were screened by bivariate analysis to allow evaluation of simple association with each of the 3 outcome variables (foals with bacteremia caused by Actinobacillus sp., enteric gram-negative organisms, and gram-positive organisms) by calculating $\chi^{2}$ tests of homogeneity as well as crude odds ratios $\left(\mathrm{OR}_{\mathrm{CR}} \mathrm{s}\right)$, associated $95 \%$ confidence intervals $(95 \% \mathrm{CI})$, and $P$ values.

Variables that met a critical $\alpha$ value of 0.25 during initial screening of bivariable associations were included for screening in the multivariable models for each of the 3 categories of microorganisms. Selection of variables for multivariable modeling with a forward stepwise multivariate logistic regression analysis was performed as described by Hosmer and Lemeshow. ${ }^{21}$ Development of the final multivariable model used a critical $\alpha$ value of 0.05 as a criterion to remain in the model. The multivariate modeling permitted evaluation of several factors simultaneously while controlling for potential confounding factors. Parameter estimates and adjusted likelihood-ratio statistics (adjusted odds ratios $\left[\mathrm{OR}_{\mathrm{ADJ}} \mathrm{s}\right]$ ) were evaluated during the modeling procedure. Adjusted $\mathrm{ORs}, 95 \% \mathrm{CI}$, and $P$ values were estimated from the final logistic regression models. The Hosmer-Lemeshow statistic was calculated for the final models to evaluate the goodness-of-fit of the data.

Differences in survival among the different types of bacteria were compared by bivariate analysis with $\chi^{2}$ tests. Differences between the outcome groups for length of hospitalization were compared by using the Mann-Whitney rank sum test. Analyses were performed on a personal computer with the statistical software SAS. ${ }^{\text {h }}$

\section{Results}

One hundred one bacteremic foals as confirmed by isolation of potentially pathogenic bacteria from blood collected antemortem and without underlying terminal congenital defects were enrolled in the study. The foals included 29 Quarter Horses, 29 Standardbreds, 7 Arabians, 6 Thoroughbreds, and 30 others (draft breeds, warmbloods, ponies, miniature horses, Morgans, and Saddlebreds). The foals ranged in age from newborn (4 foals $<1$ hour old) to 113 days, with $<10 \%$ of foals $>10$ days of age. The median age at presentation was 48 hours, with $25 \%$ of foals $<24$ hours and $75 \%<96$ hours of age at presentation. Dystocia, premature placental separation, placentitis, or illness in the mare was reported in 24\% (21/ 89 ) of foals. Thirty-one percent (30/97) of foals were reported to have been sick from birth. Nursing from the mare had been observed in $67 \%$ (64/95) of foals before presentation. A history of seizures was present in $7 \%$ (7/ $101)$, diarrhea was present in $24 \%$ (24/101), and colic was present in $13 \%(13 / 101)$. 
Table 1. Organisms cultured from the blood of 101 foals.

\begin{tabular}{|c|c|c|}
\hline Bacterial Species & $\begin{array}{l}\text { Number of } \\
\text { Foals }(\%)\end{array}$ & $\begin{array}{l}\text { Number of } \\
\text { Pure Culture } \\
(\%)\end{array}$ \\
\hline Escherichia coli $^{\mathrm{a}, \mathrm{b}}$ & $39(39)$ & $25(25)$ \\
\hline Actinobacillus sp. & $30(30)$ & 17 (17) \\
\hline Enterococcus sp. ${ }^{\mathrm{c}}$ & $14(14)$ & $8(8)$ \\
\hline Enterobacter sp. ${ }^{\mathrm{a}, \mathrm{b}}$ & $14(14)$ & $5(5)$ \\
\hline Klebsiella sp. $^{\mathrm{a}, \mathrm{b}}$ & $11(11)$ & $6(6)$ \\
\hline Streptococcus sp. ${ }^{\mathrm{c}}$ & $10(10)$ & $3(3)$ \\
\hline Salmonella sp., & $5(5)$ & $3(3)$ \\
\hline Staphylococcus sp. ${ }^{\mathrm{c}}$ & $3(3)$ & 0 \\
\hline Pseudomonas sp. ${ }^{\mathrm{b}}$ & $2(2)$ & $1(1)$ \\
\hline Listeria monocytogenes $^{\mathrm{c}}$ & $2(2)$ & $1(1)$ \\
\hline Proteus sp. ${ }^{\mathrm{a}, \mathrm{b}}$ & $1(1)$ & 0 \\
\hline Anaerobes & $6(6)$ & $2(2)$ \\
\hline
\end{tabular}

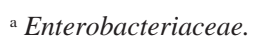

b Gram-negative enteric bacteria.

c Gram-positive bacteria.

At the time of initial examination, $51 \%$ (52/101) of foals were able to stand, $30 \%(30 / 101)$ were considered to be bright, $58 \%(59 / 101)$ were depressed, and 12\% (12/ $101)$ were comatose. Joint distention was evident in $14 \%$ $(14 / 101)$ of foals, pneumonia subsequently was confirmed in $28 \%(28 / 101)$, and septic arthritis was found in $26 \%$ $(25 / 98)$.

Forty-one percent (38/93) of foals had failure of transfer of passive immunity $(\mathrm{IgG}<400 \mathrm{mg} / \mathrm{dL})$. A sepsis score was calculated when sufficient information enabled a distinction between a score $\geq 11$ or $<11$. A discernible sepsis score was calculable in 62 bacteremic foals. Thirty foals had a sepsis score of $\leq 11$. Therefore, the sepsis score failed to predict bacteremia in $48 \%(30 / 62)$ of foals. Of the 59 foals aged $<13$ days that had a discernible sepsis score, 29 foals had a sepsis score $\leq 11$, and therefore the sepsis score failed to predict bacteremia in $49 \%(29 / 59)$ of the foals.

Actinobacillus sp. was cultured from $30 \%$ of the foals (30/101), and was the sole organism isolated in 17 (10 had A. suis and 7 had A. equuli) of these 30 foals. Eschericha coli was cultured from 39\% (39/101), with this organism being the sole isolate in 25 foals. Sixty-three percent (64/ 101) of foals had gram-negative enteric bacteremia and $30 \%(30 / 101)$ had at least 1 gram-positive organism isolated by blood culture. Six foals had growth of anaerobic organisms. Mixed infections occurred in 30\% (30/101) of foals (Table 1; Fig 1).

The overall survival rate in these bacteremic foals was $55 \%(56 / 101)$, with $56 \%$ (25/45) of deaths occurring within the 1 st 24 hours of hospitalization and $13 \%(6 / 45)$ of deaths occurring between days 10 and 20 of hospitalization. No statistically significant differences were found in survival rates among the different bacterial isolates. Foals with $A c$ tinobacillus sp. bacteremia had a slightly, but not statistically significant, greater risk $\left(\mathrm{OR}_{\mathrm{CR}}=1.9\right.$ [95\% CI: 0.8, 4.4]; $P=.14)$ of death, with a survival rate of $43 \%$ (13/ $30)$. The highest survival rate of $73 \%(11 / 15)$ was seen in foals that had only gram-positive bacteria isolated. These

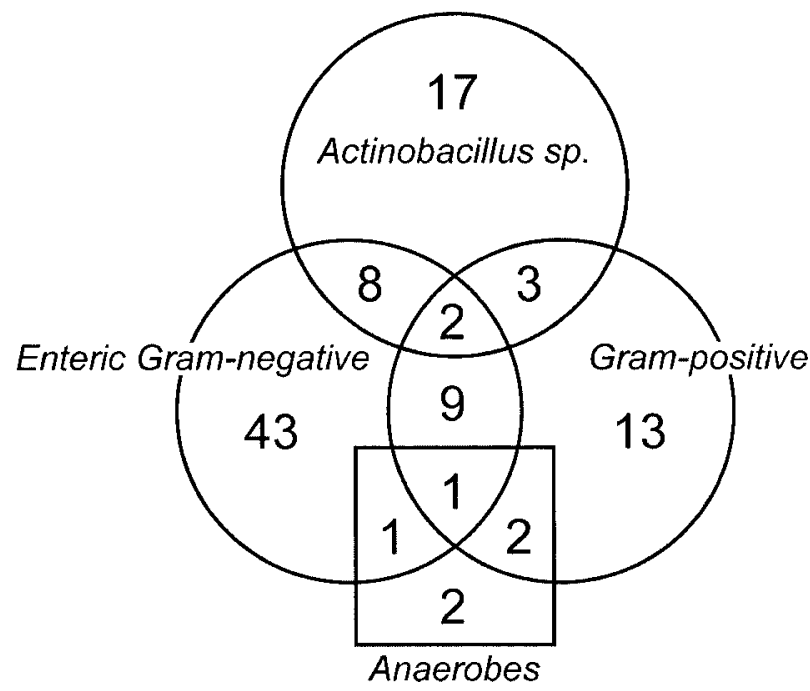

Fig 1. Venn diagram of the 3 non-mutually exclusive groups of bacteria used in statistical analysis. Although the number of foals with anaerobic infections were insufficient to perform statistical analysis on this group, it is included here so that the entire population of 101 foals is displayed.

foals had a slightly, but not significantly lower, risk of death $\left(\mathrm{OR}_{\mathrm{CR}}=0.3\right.$ [95\% CI: $\left.\left.0.09,1.1\right] ; P=.08\right)$. No statistically significant difference was found in the survival rate for foals that had a single organism cultured (55\% [38/69]), compared to those that had a mixed bacterial infection (53\% [17/32]), but foals with mixed bacterial infections had a significantly longer duration of hospitalization. Foals that had more than 1 organism isolated were hospitalized for a median of 12.5 days (95\% range: $2-42$ days), compared to 8 days (95\% range: $1-27$ days) for foals from which only a single species was isolated $(P=.04)$. Although not statistically significant, foals from which Actinobacillus sp. were cultured had the longest period of hospitalization of 12 days (95\% range: $4-26$ days; $P=.2$ ), whereas foals with a pure blood culture growth of 1 of the gram-negative enteric organisms had the shortest period of hospitalization of 6 days (95\% range: $1-23$ days; $P=.06$ ). Overall, the surviving foals were hospitalized for a median of 9 days (95\% range: $1-46$ days). There was no increased risk of failure of transfer of passive immunity $(\mathrm{IgG}>800 \mathrm{mg} / \mathrm{dL}$ compared to $\mathrm{IgG}<400 \mathrm{mg} / \mathrm{dL} ; \mathrm{OR}_{\mathrm{CR}}=1 ; 0.4,2 ; P=.8$ ) for foals with Actinobacillus sp. bacteremia compared to other foals.

Initial bivariate screening identified 12 variables significantly associated $(P<.05)$ with Actinobacillus sp. bacteremia, 8 variables associated with bacteremia involving gram-negative enteric organisms, and 3 variables were associated with gram-positive bacteremia (Table 2). Variables that met the cutoff criterion from the initial bivariate analysis (critical $\alpha<.25$ ) were screened by multivariate logistic regression. For foals with Actinobacillus sp. bacteremia, of the 26 variables that met the cutoff criterion from the initial bivariate analysis, 5 were significantly associated $(P$ $<.05)$ with the risk of Actinobacillus sp. bacteremia when adjusted for the effects of confounding variables in 
Table 2. Results of the initial bivariate screening for each of the 3 non-mutually exclusive groups of foals based on the type of bacteria cultured from the blood.

\begin{tabular}{|c|c|c|c|}
\hline Variable & $\mathrm{OR}_{\mathrm{CR}}$ & $(95 \% \mathrm{CI})$ & $P$ value \\
\hline \multicolumn{4}{|c|}{ Foals with Actinobacillus sp. bacteremia compared to other bacteremic foals } \\
\hline Evidence of illness from birth & 3 & $(1.2,7.6)$ & .02 \\
\hline Recumbent at presentation & 4.5 & $(1.7,11.5)$ & .001 \\
\hline Obtunded & 5.5 & $(1.5,20.0)$ & .005 \\
\hline Pneumonia & 2.9 & $(1.1,7.2)$ & .03 \\
\hline Venous $\mathrm{pH}\left(<7.34 \mathrm{~mm} \mathrm{Hg}^{\mathrm{a}}\right)$ & 374 & $(1.8,77,700)$ & .02 \\
\hline $\mathrm{PvCO}_{2}\left(>47.1 \mathrm{~mm} \mathrm{Hg}^{\mathrm{a}}\right)$ & 1.09 & $(1.0,1.1)$ & .002 \\
\hline Fibrinogen $(>400 \mathrm{mg} / \mathrm{dL})$ & 0.99 & $(0.99,1.00)$ & .04 \\
\hline $\mathrm{WBC}\left(<5.5 \times 10^{9}\right.$ cells $\left./ \mathrm{L}^{\mathrm{a}}\right)$ & 1.3 & $(1.1,1.6)$ & .0001 \\
\hline Segmented neutrophil count $\left(<3.3 \times 10^{9}\right.$ cells $\left./ \mathrm{L}^{\mathrm{a}}\right)$ & 1.4 & $(1.1,1.6)$ & .0001 \\
\hline Total calcium $\left(<11.5 \mathrm{mg} / \mathrm{dL}^{\mathrm{a}}\right)$ & 1.7 & $(1.2,2.5)$ & .003 \\
\hline Glucose $\left(<60 \mathrm{mg} / \mathrm{dL}^{\mathrm{a}}\right)$ & 1.01 & $(1.00,1.02)$ & .0007 \\
\hline Sepsis score $>11$ & 5.1 & $(1.4,17.9)$ & .007 \\
\hline \multicolumn{4}{|c|}{ Foals with gram-negative enteric bacteremia compared to other bacteremic foals } \\
\hline Evidence of illness from birth & 0.4 & $(0.1,0.9)$ & .03 \\
\hline Mentation score & 0.2 & $(0.04,0.9)$ & .02 \\
\hline Arterial $\mathrm{pH}\left(<7.33 \mathrm{mmHg}^{\mathrm{a}}\right)$ & $<0.00$ & $(0.0,0.6)$ & .02 \\
\hline Venous $\mathrm{pH}\left(<7.34 \mathrm{~mm} \mathrm{Hg}^{\mathrm{a}}\right)$ & $<0.00$ & $(0.0,0.13)$ & .004 \\
\hline Respiratory rate $\left(>46\right.$ breaths per minute $\left.{ }^{a}\right)$ & 1.0 & $(1.0,1.04)$ & .05 \\
\hline $\mathrm{PvCO}_{2}\left(>47.1 \mathrm{~mm} \mathrm{Hg}^{\mathrm{a}}\right)$ & 0.9 & $(0.8,1.0)$ & .0005 \\
\hline Phosphorus $\left(>5.5 \mathrm{mg} / \mathrm{dL}^{\mathrm{a}}\right)$ & 0.8 & $(0.6,1.0)$ & .03 \\
\hline $\mathrm{CK}\left(>395 \mathrm{IU} / \mathrm{L}^{\mathrm{a}}\right)$ & 0.99 & $(0.99,1.0)$ & .005 \\
\hline \multicolumn{4}{|c|}{ Foals with gram-positive bacteremia compared to other bacteremic foals } \\
\hline Evidence of illness from birth & 0.4 & $(0.1,1.1)$ & .05 \\
\hline Colic & 10.7 & $(1.0,120)$ & .04 \\
\hline $\mathrm{PaO}_{2}\left(<64.0 \mathrm{~mm} \mathrm{Hg}^{\mathrm{a}}\right)$ & $<0.00$ & $(0.0,0.0)$ & .009 \\
\hline
\end{tabular}

$\mathrm{OR}_{\mathrm{CR}}$, crude odds ratio; 95\% CI, 95\% confidence interval; $\mathrm{PvCO}_{2}$; partial pressure of carbon dioxide in venous gas; WBC; white blood cell count; $\mathrm{CK}$, creatine kinase; $\mathrm{PaO}_{2}$; partial pressure of oxygen in arterial gas.

${ }^{a}$ Median value for all foals.

the final multivariate logistic regression model. For foals with gram-negative enteric bacteremia, of the 23 variables that met the cutoff criterion in the bivariate model, 4 variables remained in the final multivariate logistic regression model (Table 3). For foals from which gram-positive organisms had been isolated, of the 17 variables that met the cutoff criterion in the bivariate model, no variables remained in the final model.

Table 3. Results of the final multivariable logistic regression model of variables associated with bacteremia caused by Actinobacillus sp. and bacteremia caused by gram-negative enteric organisms.

\begin{tabular}{|c|c|c|}
\hline Variable & $\mathrm{OR}_{\mathrm{ADJ}}(95 \% \mathrm{CI})$ & $P$ value \\
\hline \multicolumn{3}{|c|}{ Foals with Actinobacillus sp. bacteremia ${ }^{a}$} \\
\hline Evidence of illness from birth & $7 \quad(2,26)$ & .003 \\
\hline Diarrhea & $6 \quad(1,22)$ & .009 \\
\hline Recumbent on presentation & $5 \quad(1,17)$ & .006 \\
\hline Segmented neutrophil count $\left(<3.3 \times 10^{9}\right.$ cells $\left./ \mathrm{L}^{\mathrm{b}}\right)$ & $27 \quad(4,166)$ & $<.0001$ \\
\hline Band neutrophil count $\left(>0.46 \times 10^{9}\right.$ cells $\left./ L^{b}\right)$ & $4.5 \quad(1,17)$ & .02 \\
\hline \multicolumn{3}{|c|}{ Foals with gram-negative enteric bacteremia ${ }^{c}$} \\
\hline Seizures & $0.08(0.008,0.9)$ & .02 \\
\hline Evidence of illness from birth & $0.3(0.1,0.9)$ & .04 \\
\hline $\mathrm{PvCO}_{2}\left(>47.1 \mathrm{~mm} \mathrm{Hg}^{\mathrm{b}}\right)$ & $0.2 \quad(0.08,0.55)$ & .001 \\
\hline $\mathrm{PaCO}_{2}\left(>42.8 \mathrm{~mm} \mathrm{Hg}^{\mathrm{b}}\right)$ & $0.3(0.07,0.095)$ & .04 \\
\hline
\end{tabular}

OR ${ }^{\mathrm{ADJ}}$, adjusted odds ratio; 95\% CI, 95\% confidence interval; $\mathrm{PvCO}_{2}$; partial pressure of carbon dioxide in venous gas; $\mathrm{PaCO}$; partial pressure of carbon dioxide in arterial gas.

${ }^{a}$ Homser-Lemeshow goodness of fit statistic; $\chi^{2}=9.3, P=.31$.

${ }^{\mathrm{b}}$ Median value for all foals.

${ }^{\mathrm{c}}$ Homser-Lemeshow goodness of fit statistic; $\chi^{2}=2.8, P=.59$. 


\section{Discussion}

Foals with Actinobacillus sp. bacteremia were more likely to be ill from birth, to have diarrhea, to be recumbent at presentation, to have a sepsis score $>11$, to be obtunded, to have pneumonia, to have a lower segmented neutrophil count, and to have a higher band neutrophil count than other bacteremic foals. However, the overall survival rate for foals with Actinobacillus sp. bacteremia was similar to that of foals with bacteremia due to infection with either gram-negative enteric or gram-positive bacteria. Foals with bacteremia caused by enteric gram-negative organisms were unlikely to be ill from birth, and had lower frequencies of seizures and hypercapnia. The type of organism isolated from the blood of foals did not significantly influence mortality rate.

Bacterial septicemia is the leading cause of morbidity and mortality in equine neonates., ${ }^{2,7,22-27}$ Survival rates reported over the last 10 years in retrospective studies of septicemic foals are highly variable, ranging between 10 and 70\%, ${ }^{5-7,24}$ and comparisons among studies are difficult because of differences in case definition. Septicemia is a presumptive diagnosis, based upon the overall clinical impression gained from a combination of clinical variables, and for neonatal foals the sepsis score system described by Brewer and Koterba ${ }^{20}$ often is used. In contrast, the diagnosis of bacteremia requires identification of the etiologic agent by bacteriologic culture of blood and is independent of the severity of the resulting illness. Our study is based on a definitive antemortem diagnosis of bacteremia as demonstrated by a positive culture in blood collected at the time of admission of potentially septicemic foals.

When the etiology of equine neonatal septicemia is of bacterial origin, the likelihood of a positive blood culture being obtained may be dependent on the concentration of bacteria in the bloodstream, the immune status of the foal, previous antibiotic therapy, and the number of blood cultures performed. However, these assumptions have never been formally investigated. A positive culture may not necessarily be obtained from a neonate with severe sepsis, and some confirmed bacteremic foals may not demonstrate clinical signs consistent with septicemia. Therefore, the distinction between presumptive septicemia and confirmed bacteremia should be maintained because they define 2 different but overlapping populations.

The diagnosis of bacteremia is unequivocal, whereas that of septicemia is less distinct and is subject to interpretation. This difference results in confusion and difficulty in comparing conclusions drawn from previous studies of foal septicemia. The diagnosis of bacteremia can have a specificity of up to $100 \%$ with careful sampling technique, which can exclude bacterial contamination. The sensitivity of the diagnosis of bacteremia is difficult to determine, and depends on sampling and microbiological processing techniques, concentration of bacteria in the bloodstream at the time of sample collection, immunologic status of the animal, and previous or current antibiotic therapy. Several reports describe success in obtaining positive antemortem blood culture from septicemic foals diagnosed by various means. ${ }^{2,4,5,7,24,27}$ Little information is available to correlate the incidence of bacteremia with the severity of the clini- cally observed sepsis. Although useful in the assessment of the severity of illness, we found sepsis score (performed at admission) to be an unreliable indicator of bacteremia.

The present study of bacteremic foals differs from many previous studies of the risk factors and prognosis associated with equine neonatal septicemia in several ways. Previous studies included combinations of foals with bacteremia confirmed by antemortem blood culture, those in which bacterial infection was confirmed by culture of postmortem specimens, and those in which bacteriologic cultures were obtained from joints, umbilicus, peritoneal fluid, or pleural fluid. Cases also were included based on evidence of multisystemic infectious disease or sepsis score $>11 .^{2,4,6,7,24}$ Such a heterogeneous mix of patient populations makes any comparison of mortality, prognostic indicators, or causation among studies difficult. Our study is specific in its inclusion criteria containing 101 foals with a definitive diagnosis of bacteremia confirmed by positive antemortem blood culture, and therefore potentially encompasses a different patient population from previous investigations.

In our hospital, blood cultures are performed routinely on all potentially septicemic foals at admission. In the majority of cases, only a single sample is obtained for culture, but if clinical deterioration occurs, or the foal fails to respond to antimicrobial therapy, additional cultures may be performed. This study was based on results of a single blood culture collected at the time of presentation. In human medicine, it is recommended that 3 or 4 blood cultures be collected 1 hour apart to maximize the chances of detecting bacteremia. However, a positive result is obtained from the 1 st culture in $92 \%$ of cases, ${ }^{28}$ indicating the high sensitivity of performing only a single blood culture. In foals, the success rate of obtaining a positive culture is variable among different studies. Koterba et $\mathrm{al}^{2}$ reported a $60 \%$ positive culture rate, Wilson and Madigan ${ }^{4}$ found that $81 \%$ of confirmed septic foals had positive blood culture results, and Gayle et $\mathrm{al}^{7}$ found $83 \%$ of septicemic foals to have bacterial growth on blood culture. Brewer and Koterba ${ }^{5}$ found a $12 \%$ false-negative rate, and 23 of 186 foals that had negative blood cultures were found to have disseminated sepsis at postmortem examination. The present study did not attempt to determine the false-negative rate of confirmation of bacteremia. Data were compiled from medical records of foals from which a positive blood culture was obtained in an effort to determine the effects of different types of bacteria. As a result, information from foals with false-negative blood cultures and undetermined etiologies was not considered useful in addressing our primary objectives. Ideally, an investigation performed in parallel to the present study comparing variables between the antemortemconfirmed bacteremic foals and septicemic foals that had negative blood culture results would have provided a largescale statistical comparison between the 2 groups.

Blood culturing is considered a routine procedure in most neonatal intensive care units, but the differences in survival rates for the 2 overlapping populations of septicemic and bacteremic foals has not been conclusively determined. Gayle et $\mathrm{al}^{7}$ found similar survival rates between foals with positive antemortem microbiologic culture and septicemic foals diagnosed by evidence of multisystemic infection or 
sepsis score $>11$ that did not have growth of bacteria on antemortem blood cultures.

Previous investigations have limited inclusion criteria to the age of the foal. Although most of foals in this study were neonates, with $75 \%$ of the foals presenting at $<96$ hours of age, there was no age restriction. Any foal for which the attending clinician considered a blood culture warranted, and from which a positive result was obtained, was included in the analysis. Only $10 \%$ of the foals in this study were $>10$ days of age and the oldest foal was 113 days old.

The weighted sepsis scoring system designed by Brewer and Koterba ${ }^{20}$ is based on historical, clinical, and laboratory variables, and uses a score $>11$ as a predictor of sepsis in foals $<13$ days of age. The calculations of Brewer and Koterba $^{20}$ were based on a group of septic foals composed of a combination of septicemic foals (defined as the presence of a positive antemortem blood culture or infection at 2 or more locations confirmed at postmortem examination) and focally infected patients (based on criteria of a positive culture of a symptomatic body system, infection at postmortem examination, or obviously infected areas with positive cytologic examination). The weightings used in the development of the scoring system were based on the authors' clinical judgment and data from 19 cases. No statistical analysis was used in the creation of the weightings. ${ }^{20}$ The sensitivity and specificity of this scoring system were reported to be $94 \%$ and $86 \%$, respectively, in predicting the presence or absence of septicemia when tested on 209 neonatal foals $\left(<13\right.$ days of age), ${ }^{29}$ but these findings were not confirmed in other hospital settings. Other investigators have found this sepsis scoring system to be less reliable at predicting septicemia at their institutions. ${ }^{30}$ In the present study, of the 62 bacteremic foals that had a calculable sepsis score, 30 foals had scores $\leq 11$. Therefore, the sepsis score had a sensitivity of only $52 \%$ in predicting bacteremia. Restricting the population to foals $<13$ days did not improve the sensitivity of the test. The sepsis score was designed to predict septicemia and not necessarily bacteremia, but it has been advocated as a tool to determine the necessity of antibiotic therapy in neonatal foals while blood culture results are pending..$^{20}$ Because of the poor sensitivity of the sepsis score for predicting bacteremia, our results emphasize the importance of prompt initiation of antibiotic therapy of any potentially bacteremic foal, even if pooled clinical variables are not suggestive of septicemia at the initial examination. Antibiotic therapy is recommended from the time of presentation rather than waiting for bacterial growth on blood culture or clinical deterioration to a sepsis score $>11$. The sepsis scoring system seems to be useful in predicting sepsis, but not necessarily in predicting bacteremia. This further emphasizes the potential difference in populations of septicemic versus bacteremic foals. The definition of bacteremia does not include an assessment of severity of observed clinical signs. Some bacteremic foals that show minimal clinical derangements may recover uneventfully with appropriate antimicrobial therapy. It is logical to predict that bacteremic foals showing signs of severe sepsis would have a worse prognosis.

Survival rates reported in previous retrospective studies of septicemic foals are variable. The following survival rates were reported: 45\% (29/65; Gayle et $\left.\mathrm{al}^{7}\right), 70 \%$ (17/ 24; Raisis et $\mathrm{al}^{6}$ ), 45\% (29/65; Brewer and Koterba ${ }^{5}$ ), 26\% $\left(10 / 38\right.$; Koterba et $\mathrm{al}^{2}$ ), and $10 \%$ (Hoffman et $\mathrm{al}^{24}$ ). Comparisons are difficult because of differences in case definitions, ages of the foals, financial situations of owners, and management strategies available at clinics in different geographic locations. Our overall survival rate for bacteremic foals of 54\% (55/101) is comparable to survival rates observed in other studies of septicemic foals. Although foals with Actinobacillus bacteremia had a slightly lower survival rate, and foals with gram-positive infections had a slightly higher survival rate, no statistically significant difference was found in survival rates of the foals based on the type of bacteria isolated from their blood. Other studies also found a tendency for increased survival in foals with grampositive bacterial septicemia. ${ }^{5}$ Earlier reports indicate a higher mortality $(81 \%)$ in foals with mixed bacterial infection compared to the mortality rate $(57 \%)$ in foals from which a single bacterial species was isolated. ${ }^{27}$ Our results do not agree with those of previous studies. We found no difference between the survival rates of foals from which a single isolate was cultured compared to those that had a mixed bacterial infection, but foals with a mixed bacterial infection had a significantly prolonged length of hospitalization.

Although we did not find the age of the foal at presentation to be influenced by the type of bacterial isolate, we were able to confirm that foals with Actinobacillus sp. bacteremia were more likely to be sick from birth. This raises the question of in utero-derived infection or early postnatal infection. The high frequencies of recognized illness from birth could not be explained by sickness of the mare or dystocia. Foals with Actinobacillus sp. bacteremia were just as likely to stand and nurse the mare, with no difference in IgG concentrations at presentation compared to other bacteremic foals. At presentation, foals with actinobacillosis were more likely to be recumbent, and based on bivariate analysis they were more likely to have a sepsis score $>11$ and be obtunded. Our study indicates that although these foals may appear sicker at presentation, with appropriate treatment survival rates are not statistically different from those of other bacteremic foals.

Foals with enteric gram-negative bacteremia had a significantly lower frequency of illness in the time period immediately after birth, they were less likely to have seizures, or less likely to have high arterial or venous $\mathrm{PCO}_{2}$ than foals with Actinobacillus sp. or gram-positive bacteremia. Although no variables remained in the logistic regression model for gram-positive bacteria, we did find an increased frequency of these bacterial isolates compared to studies performed a decade ago, when isolation of pure cultures of gram-positive organisms was considered to be an infrequent occurrence. ${ }^{1,2,5,6,18}$ The frequency of gram-positive infection was $30 \%(30 / 101)$ in our study, with $57 \%$ (17/30) of foals with gram-positive infections having a mixed bacterial infection. The results of previous studies are quite variable. Wilson and Madigan ${ }^{4}$ found that gram-positive and anaerobic isolates were always part of a mixed gram-negative infection. Paradis ${ }^{27}$ found gram-positive organisms in $18 \%$ (27/153) of isolates, with $89 \%$ (24/27) of gram-positive isolates being part of a mixed bacterial infection. More re- 
cently, Marsh and Palmer ${ }^{9}$ found a high incidence of grampositive infections, in which case a single gram-positive isolate was obtained from $34 \%(49 / 155)$ of bacteremic foals. Although the prevalence of gram-positive infections and the frequency of these organisms being cultured as a single isolate seems to be increasing, we found only $13 \%$ $(13 / 101)$ of bacteremic foals in this study to have a singleisolate gram-positive infection.

Potential pitfalls of our study include its retrospective nature, dependence on the accuracy and completeness of the medical record, and also limitations on the extensiveness of the initial diagnostic workup because of the unavailablity of some diagnostic tests for after-hours admissions and financial limitations imposed by some owners. Lack of historical data regarding pre- and periparturition events and frequent absence of fibrinogen concentration and blood gas analysis made accurate calculation of the sepsis score impossible in a large number of cases. Therefore, a sepsis score was only recorded if it definitively differentiated between foals with a sepsis score $\leq 11$ or $>11$. A range in severity of signs of sepsis was observed in the bacteremic foals included in the modeling procedures. A small number of blood cultures were obtained immediately after birth from foals delivered from hospitalized sick mares, from relief of dystocia, or after caesarian delivery. These foals all were given antibiotics intravenously immediately after birth, with the majority making an uneventful recovery. Bacteremia in these foals may have been a transient event, but it cannot be determined whether lack of antimicrobial therapy would have resulted in a different outcome for these individuals. Financial limitations and differences in clinician treatment strategies and criteria for discharge influence outcome variables such as length of hospitalization and survival rate, but probably had little effect on the overall comparison among the different categories of organisms.

As a retrospective, single-site study, it is uncertain whether our results can be extrapolated to other institutions, geographic regions, or countries. The width of confidence intervals and power of the study could only be improved by inclusion of a greater number of foals. Based on the results obtained, a large-scale, prospective, multicenter study is warranted.

In agreement with anecdotal reports and our hypothesis, foals that present with a combination of being ill from birth, having diarrhea, being recumbent at presentation, and having a low segmented neutrophil count and high band neutrophil count should be considered likely to have Actinobacillus sp. bacteremia. Given that actinobacilli in some regions have different antimicrobial susceptibilities when compared to enteric gram-negative organisms, ${ }^{9}$ early differentiation of foals with Actinobacillus sp. bacteremia will allow the immediate institution of appropriate antibiotic therapy. Although the bacterial isolate had no influence on survival rate, early diagnosis and effective antimicrobial therapy may help to decrease the morbidity and mortality associated with equine neonatal bacteremia. Results of this study should improve the veterinary internist's ability to predict the causative organism before results of blood culture are available.

\section{Footnotes}

a BBL Septic-Check Blood Collection Bottles, $70 \mathrm{~mL}$ Trypticase Soy Broth with sodium polyanetholsulfonate (SPS) and $\mathrm{CO}_{2}$ and $70 \mathrm{~mL}$ Columbia Broth with SPS and $\mathrm{CO}_{2}$, Becton Dickinson Microbiology Systems, Beckon Dickinson and Company, Cockeysville, MA

${ }^{\mathrm{b}}$ Vacutainer, Becton Dickinson, Parsipany, NJ

c Coulter Counter Model S-plus IV, Coulter Electronics Inc, Hialeah, FL, until March 23, 2000; thereafter Cell-Dyn 3500R, Abbott Diagnostics, Abbott Park, IL

${ }^{\mathrm{d}}$ DACO-Dart Reagent System, Coulter Electronics Inc, Hialeah, FL, until November 1, 1994; thereafter Boehringer Mannheim/Hitachi (Roache) 911 System, Roache, Indianapolis, IL

${ }^{\mathrm{e}}$ Concentration immunoassay technology (CITE), Idex, Portland, ME

${ }^{\mathrm{f}}$ ACL200, Instrumentation Laboratories, Lexington, MA

g IL1307, Instrumentation Laboratories, Lexington, MA

${ }^{\text {h }}$ PC SAS, SAS Institute Inc, Cary, NC

\section{Acknowledgments}

We would like to acknowledge Leia Hill for her assistance in some of the initial data entry and Alan E. Lawrence, Kelli A. Wertenberger, and Teri L. West from the Veterinary Microbiology Laboratory for their assistance.

\section{References}

1. Platt H. Septicaemia in the foal. A review of 61 cases. Br Vet $\mathbf{J}$ 1973;129:221-229.

2. Koterba AM, Brewer BD, Tarplee FA. Clinical and clinicopathological characteristics of the septicaemic neonatal foal: Review of 38 cases. Equine Vet J 1984;16:376-382.

3. Martens RL, Auer JA, Carter GK. Equine pediatrics: Septic arthritis and osteomyelitis. J Am Vet Med Assoc 1986;188:582-585.

4. Wilson WD, Madigan JE. Comparison of bacteriologic culture of blood and necropsy specimens for determining the cause of foal septicemia: 47 cases (1978-1987) [published erratum appears in J Am Vet Med Assoc 1990;196:438]. J Am Vet Med Assoc 1989;195:1759_ 1763.

5. Brewer BD, Koterba AM. Bacterial isolates and susceptibility patterns in foals in a neonatal intensive care unit. Compend Cont Educ Pract Vet 1990;12:1773-1781.

6. Raisis AL, Hodgson JL, Hodgson DR. Equine neonatal septicaemia: 24 cases. Aust Vet J 1996;73:137-140.

7. Gayle JM, Cohen ND, Chaffin MK. Factors associated with survival in septicemic foals: 65 cases (1988-1995). J Vet Intern Med 1998;12:140-146.

8. Raisis AL, Hodgson JL, Hodgson DR. Serum gentamicin concentrations in compromised neonatal foals. Equine Vet J 1998;30:324328.

9. Marsh PS, Palmer JE. Bacterial isolates from the blood and their susceptibility patterns in critically ill foals: 543 cases (1991-1998). J Am Vet Med Assoc 2001;218:1608-1610.

10. Jang SS, Biberstein EL, Hirsh DC. Actinobacillus suis-like organisms in horses. Am J Vet Res 1987;48:1036-1038.

11. Nelson KM, Darien BJ, Konkle DM, et al. Actinobacillus suis septicaemia in two foals. Vet Rec 1996;138:39-40.

12. Radostits OM, Gay CC, Blood DC, et al. Veterinary Medicine-A Textbook of the Diseases of Cattle, Sheep, Pigs, Goats and Horses, 9th ed. London: WB Saunders; 2000:942-943.

13. Koterba AM, House JK. Neonatal infection. In: Smith BP, ed. Large Animal Internal Medicine, 2nd ed. St Louis, MO: Mosby; 1996: $344-353$. 
14. Cottew GS. Shigella equirulis infection in a foal. Aust Vet J 1952:302-306.

15. Rycroft AN, Woldeselassie A, Gordon PJ, et al. Serum antibody in equine neonatal septicaemia due to Actinobacillus equuli. Vet Rec 1998;143:254-255.

16. Jubb KVF, Kennedy PC, Palmer N. In: Jubb K, Kennedy P, Palmer N, eds. Pathology of Domestic Animals, 4th ed. San Diego, CA: Academic Press; 1993:419.

17. Bredin KJ. The clinical approach to some foal diseases in an Australian stud practice. Ir Vet J 1973;27:63-68.

18. Whitwell KE. Investigations into fetal and neonatal loss in the horse. Vet Clin North Am 1980;2:313-331.

19. Brewer BD. Neonatal infection. In: Koterba A, ed. Equine Clinical Neonatology. Philidelphia, PA: Lea \& Febiger; 1990:295-316.

20. Brewer BD, Koterba AM. Development of a scoring system for the early diagnosis of equine neonatal sepsis. Equine Vet J 1988;20 $18-22$.

21. Hosmer DW, Lemeshow S. Applied Logistic Regression. New York: John Wiley \& Sons; 1989.

22. Baker SM, Drummond WH, Lane TJ, et al. Follow-up evaluation of horses after neonatal intensive care. J Am Vet Med Assoc 1986; 189:1454-1457.
23. Morris DD, Moore JN. Tumor necrosis factor activity in serum from neonatal foals with presumed septicemia. J Am Vet Med Assoc 1991;199:1584-1589.

24. Hoffman AM, Staempfli HR, Willan A. Prognostic variables for survival of neonatal foals under intensive care. J Vet Intern Med 1992; 6:89-95.

25. Freeman L, Paradis MR. Evaluating the effectiveness of equine neonatal care. Vet Med 1992;87:921-926.

26. Cohen ND. Causes of and farm management factors associated with disease and death in foals. J Am Vet Med Assoc 1994;204:16441651.

27. Paradis MR. Update on neonatal septicemia. Vet Clin North Am 1994;10:109-135.

28. Lyman J. Use of blood cultures in the emergency department. Ann Emerg Med 1986;15:308.

29. Brewer BD, Koterba AM, Carter RL, et al. Comparison of empirically developed sepsis score with a computer generated and weighted scoring system for the identification of sepsis in the equine neonate. Equine Vet J 1988;20:23-24.

30. Corley KTT, Furr MO. Evaluation of the sepsis score for neonatal septicemia. 38th British Equine Veterinary Association Annual Congress, 1999. 